\title{
AN EXPANSION FORMULA FOR DIFFERENTIAL EQUATIONS
}

\author{
BY KUO-TSAI CHEN
}

Communicated by Deane Montgomery, March 2, 1962

Denote by $z=\left(z^{1}, \cdots, z^{n}\right)$ the coordinates of the $n$-dimensional complex space. If $m=\left(m_{1}, \cdots, m_{n}\right)$ is an $n$-tuple of nonnegative integers, then we write $z^{m}=\left(z^{1}\right)^{m_{1}} \cdots\left(z^{n}\right)^{m_{n}}$. Any polycylinder mentioned in this paper will have its center at the origin.

Let $f(t, z)$ depend on the complex variables $z$ and a parameter $t$ over a measurable set $I$ in a measure space with measure $\mu$. We say that $f(t, z)$ is dominatedly integrable over $I$ for $z$ in a polycylinder $U$, if the following conditions are satisfied:

(a) For a.e. (almost every) value of the parameter $t$ in $I, f(t, z)$ is holomorphic in $U$.

(b) The expansion $\sum a_{m}(t) z^{m}$ of $f(t, z)$ is such that each coefficient $a_{m}$ is integrable over $I$.

(c) The series $\sum \int_{I}\left|a_{m}(t)\right| d \mu z^{m}$ converges in $U$.

It can be easily shown that $F(z)=\int_{I} f(t, z) d \mu$ is holomorphic in $U$, and this integration commutes with partial differentiation with respect to $z$.

Definition. We say that $A(t)=\sum a^{i}(t, z) \partial / \partial z^{i}, t$ being a real variable, is a t.d.i.t. (time dependent infinitesimal transformation) if there exists a polycylinder $U$ such that, for $z$ in $U$, each $a^{i}(t, z)$ is dominatedly integrable in the sense of Lebesgue over any finite interval $I$.

Definition. For $f(z)$ holomorphic about the origin, we define $T^{*}\left(A ; t, t_{0}\right) f$, or simply $T^{*}(t) f$, to be the sum function of the series

$$
T_{0}^{*}(t) f+\cdots+T_{r}^{*}(t) f+\cdots,
$$

where $T_{0}^{*}(t) f=f$ and, for $r>0, T_{r}^{*}(t) f=\int_{t_{0}}^{t} T_{r-1}^{*}(s) A(s) f d s$.

Our main purpose is to prove the formula

$$
\left(T^{*}(t) f\right)\left(z_{0}\right)=f\left(T(t) z_{0}\right),
$$

where $z=T(t) z_{0}$ denotes the solution of the system of differential equations $d z^{i} / d t=a^{i}(t, z)$ with the initial condition $z\left(t_{0}\right)=z_{0}$.

By direct computation, it is verified that

$$
\frac{d}{d t} \sum_{i=0}^{r}\left(T_{i}^{*}(t) f\right)\left(T_{r-i}^{*}(t) g\right)=d T_{r}^{*}(t)(f g) / d t .
$$

Consequently, if $T^{*}(t) f$ and $T^{*}(t) g$ both converge absolutely in a 
neighborhood of the origin, then

$$
T^{*}(t)(f g)=\left(T^{*}(t) f\right)\left(T^{*}(t) g\right) .
$$

In other words, $T^{*}(t)$ can be looked upon as an endomorphism of the ring of the functions holomorphic about the origin.

For $f(z)=\sum a_{m} z^{m}$ and $g(z)=\sum b_{m} z^{m}$ holomorphic about the origin, we write $f \ll g$ when $\left|a_{m}\right| \leqq b_{m}$ for all $m$. For two t.d.i.t. $A(t)$ and $B(t)$, we write $A(t) \ll B(t)$ when $A(t) z^{i} \ll B(t) z^{i}, i=1, \cdots, n$, for a.e. value of $t$.

We obtain, in a rather straightforward manner, the following result:

THEOREM 1. If $A(t) \ll B(t)$ and if $f \ll g$, then, for $t>t_{0}$, the existence of $T^{*}\left(B ; t, t_{0}\right) g$ in a polycylinder $U$ will imply that of $T^{*}\left(A ; t, t_{0}\right) f$ in the same polycylinder. Moreover

$$
T^{*}\left(A ; t, t_{0}\right) f \ll T^{*}\left(B ; t, t_{0}\right) g .
$$

It is known that, if $X$ is an infinitesimal transformation (holomorphic about the origin and independent on the time $t$ ), then $T^{*}\left(X ; t, t_{0}\right) f=\left(\exp \left(t-t_{0}\right) X\right) f$ exists. By constructing a suitable $X$ with $A(t) \ll X$, we are led to the next proposition:

THEOREM 2. If $\left|A(t) z^{i}\right| \leqq M$ for a.e. value of $t$ and for $z$ in a given polycylinder of radius $R$, then $T^{*}(t) f$ exists and is holomorphic about the origin provided the number $\left|t-t_{0}\right| M / R$ is sufficiently small.

Hereafter we shall assume that $A(t)$ satisfies the conditions of the above theorem. For our purpose, this assumption is almost superficial, because $T^{*}\left(t_{1}\right)$ remains the same when $A(t)$ is subject to any alteration for values of $t$ beyond the interval between $t_{0}$ and $t_{1}$.

Let $z_{0}$ be any point sufficiently close to the origin. Denote by $\alpha_{z_{0}}(t)$ the path given by

$$
z\left(\alpha_{z_{0}}(t)\right)=\left(T^{*}(t) z\right)\left(z_{0}\right) .
$$

Since $T^{*}(t)$ is an endomorphism of the ring of the holomorphic functions about the origin, we obtain, for any polynomial $f$ of $z$,

$$
f\left(\alpha_{z_{0}}(t)\right)=\left(T^{*}(t) f\right)\left(z_{0}\right) \text {. }
$$

By passing to limit, the above identity also holds for any function $f$ holomorphic about the origin. It follows that

$$
\begin{aligned}
d z^{i}\left(\alpha_{z_{0}}(t)\right) / d t & =\left(T^{*}(t) A(t) z^{i}\right)\left(z_{0}\right) \\
& =\left(T^{*}(t) a^{i}(t, z)\right)\left(z_{0}\right) \\
& =a^{i}\left(t, z\left(\alpha_{z_{0}}(t)\right)\right) .
\end{aligned}
$$


In short, $\alpha_{z_{0}}(t)=T(t) z_{0}$. Hence (1) follows from (2).

In order to indicate some application of the above results, we consider again the system of differential equations $d z^{i} / d t=a^{i}(t, z)$ represented by the t.d.i.t. $A(t)$. Denote by $a_{r}^{i}(t, z)$ the component of degree $r$ in the expansion of the function $a^{i}(t, z)$ about the origin, and write $A_{r}(t)=\sum_{i=1}^{n} a_{r}^{i}(t, z) \partial / \partial z^{i}$. Then we study local properties of the solutions of the system of differential equations through the power series expansion of $T^{*}(t) z$ in $z$.

EXAmple. Consider the system of the differential equations

$$
\begin{aligned}
& d x / d t=-y+f(x, y), \\
& d y / d t=x+g(x, y),
\end{aligned}
$$

where $f$ and $g$ are holomorphic with their respective expansions of order at least 2 about $(x, y)=(0,0)$. Set $z=e^{-i t}(x+i y)$ and $\bar{z}=e^{i t}(x-i y)$. Then

$$
\begin{aligned}
& d z / d t=e^{-i t} h\left(e^{i t} z, e^{-i t \bar{z}}\right), \\
& d \bar{z} / d t=e^{i t} \bar{h}\left(e^{-i t} \bar{z}, e^{i t} z\right),
\end{aligned}
$$

where $h(x+i y, x-i y)=f(x, y)+i g(x, y)$, and $\bar{h}$ is obtained from $h$ by replacing, in the power series expansion of $h$, each coefficient by the conjugate. Corresponding to (4), we have the t.d.i.t.

$$
A(t)=e^{-i t} h\left(e^{i t} z, e^{-i t \bar{z}}\right) \partial / \partial z+e^{i t} h\left(e^{-i t} \bar{z}, e^{i t} z\right) \partial / \partial \bar{z} .
$$

It is clear that $A_{0}(t)=A_{1}(t)=0$ and $\int_{0}^{2 \pi} A_{2}(t) d t=0$. The first nonvanishing component of the power series expansion of $T^{*}(A ; 2 \pi, 0) z-z$ has degree 3 and is equal to

$$
\left\{\int_{0}^{2 \pi} A_{3}(s) d s+\int_{0}^{2 \pi} \int_{0}^{s} A_{2}(s) A_{2}\left(s^{\prime}\right) d s d s^{\prime}\right\} z
$$

Write

$$
\begin{aligned}
A_{3}(t)= & e^{-i t}\left(\cdots+k e^{i t} z^{2} \bar{z}+\cdots\right) \partial / \partial z \\
& +e^{i t}\left(\cdots+\bar{k} e^{-i t} z \bar{z}^{2}+\cdots\right) \partial / \partial z .
\end{aligned}
$$

Then $\int_{0}^{2 \pi} A_{3}(s) d s z=2 \pi k z^{2} \bar{z}$. On the other hand, we write

$$
\begin{aligned}
A_{2}(t)= & e^{-i t}\left(a e^{2 i t} z^{2}+2 b z \bar{z}+c e^{-2 i t} \bar{z}^{2}\right) \partial / \partial z \\
& +e^{i t}\left(\bar{a} e^{-2 i t} \bar{z}^{2}+2 \bar{b} z \bar{z}+\bar{c} e^{2 i t} z^{2}\right) \partial / \partial \bar{z}
\end{aligned}
$$

and obtain

$$
\int_{0}^{2 \pi} \int_{0}^{8} A_{2}(s) A_{2}\left(s^{\prime}\right) d s d s^{\prime} z=-4 \pi i z^{2} \bar{z}(2 b \bar{b}-a b+c \bar{c} / 3) .
$$


Therefore

$$
T^{*}(A ; 2 \pi, 0) z=z+2 \pi z^{2} \bar{z}(k-2 i(2 b \bar{b}-a b+c \bar{c} / 3))+\cdots .
$$

Now we restrict ourselves to the case where the system (3) is real. The solution of the system with the initial condition that $x=x_{0}$, $y=y_{0}$ when $t=0$, can be given through the formula

$$
x+i y=e^{i t_{z}}=e^{i t}\left(T^{*}(A ; t, 0) z\right)_{z=z_{0}}
$$

provided $z_{0}=x_{0}+i y_{0}$ is sufficiently close to 0 . Let $\left(x_{1}, y_{1}\right)$ be the point reached by the integral curve when $t=2 \pi$. Then

$$
\begin{aligned}
x_{1}+i y_{1} & =\left(T^{*}(A ; 2 \pi, 0) z\right)_{z=z_{0}} \\
& =z_{0}+2 \pi z_{0}^{2} \bar{z}_{0}(k-2 i(2 b \bar{b}-a b+c \bar{c} / 3))+\cdots .
\end{aligned}
$$

If $r_{0}^{2}=x_{0}^{2}+y_{0}^{2}$, then

$$
\begin{aligned}
x_{1}^{2}+y_{1}^{2} & =z_{0} \bar{z}_{0}+4 \pi(\operatorname{Re} k-2 \operatorname{Im}(a b))\left(z_{0} \bar{z}_{0}\right)^{2}+\cdots \\
& =r_{0}^{2}+K r_{0}^{4}+\cdots .
\end{aligned}
$$

Hence we conclude that the integral curve of the autonomous system (3), after a time lapse of $2 \pi$, will carry every point in a sufficiently small neighborhood of $(0,0)$ in the real $(x, y)$-plane farther away or closer to $(0,0)$ according as $K>0$ or $K<0$.

\section{BIBLIOGRAPHY}

1. K. T. Chen, Formal differential equations, Ann. of Math. 73 (1961), 110-133. 278.

2. - Decomposition of differential equations, Math. Ann. 146 (1962), 263-

3. S. Lefschetz, Differential equations: geometric theory, Interscience, New York, 1957.

4. J. K. Hale, Integral manifolds of perturbed differential systems, Ann. of Math. 73 (1961), 496-531.

INSTITUTE FOR ADVANCED STUdy 